\begin{tabular}{|c|c|}
\hline Title & Evolving conductive polymer neural networks on wetware \\
\hline Author(s) & $\begin{array}{l}\text { A kai-Kasay a, Megumi; Hagiwara, Naruki; Hikita, W ataru; Okada, Masaru; Sugito, Y asumasa; Kuwahara, Y uji; A sai, } \\
\text { Tetsuya }\end{array}$ \\
\hline Citation & $\begin{array}{l}\text { Japanese Journal of A pplied Physics(JJA P), 59(6), } 060601 \\
\text { https://doi.org/10.35848/1347-4065/ab8e06 }\end{array}$ \\
\hline Issue Date & $2020-06-01$ \\
\hline Doc URL & http:/hdl .handle.net/2115/81643 \\
\hline Rights & (O2020 The Japan Society of A pplied Physics \\
\hline Type & article (author version) \\
\hline File Information & Comp_Neur_Net_Polym_Wire_Main_text_F.pdf \\
\hline
\end{tabular}

Instructions for use 


\title{
Evolving Conductive Polymer Neural Networks on Wetware
}

\author{
Megumi Akai-Kasaya ${ }^{1,2}$, Naruki Hagiwara ${ }^{1}$, Wataru Hikita ${ }^{1}$, Masaru Okada ${ }^{1}$, \\ Yasumasa Sugito $^{1}$, Yuji Kuwahara ${ }^{1}$ and Tetsuya Asai ${ }^{2}$ \\ ${ }^{1}$ Graduate School of Engineering, Osaka University, 2-1 Yamadaoka, Suita, Osaka \\ 565-0871, Japan \\ ${ }^{2}$ Faculty of Information Science and Technology, Hokkaido University, Kita 14, \\ Nishi 9, Kita-ku, Sapporo 060-0814, Japan
}

\begin{abstract}
Neural networks in the brain are structured in three-dimensional (3-D) space, and the networks evolve through development and learning, whereas two-dimensional (2-D) crossbars have essentially been optimized for a fully connected neural network, which results in a significant increase in unused memristors. Here, we present a prototype of molecular neural networks on wetware consisting of a space-free synaptic medium immersed in monomer solution. In the medium, conductive polymer wires are grown between multiple electrodes through learning only when necessary, i.e., no polymer wire is pre-placed, unlike present 2-D crossbar devices. Through experiments, we found the necessary growth conditions for synaptic polymer wires. We first demonstrated the learning of simple Boolean functions and then data-encoding tasks by using our system comprising the synaptic media and their external controllers. These results are valuable for expanding the concept of space-free synapse development, i.e., extending our 2-D synaptic media to $3-\mathrm{D}$ is possible in principle.
\end{abstract}

\section{Introduction}

An artificial neural network (ANN) is one of the key components in recent artificial intelligence (AI) based on deep learning technologies, and is capable of performing a variety of computational tasks such as cognition, prediction, optimization, and intuitive representation ${ }^{1,2}$. Present digital computers based on von Neumann architectures are, however, not well-adapted to perform ANN computing because of the physical separation of the arithmetic and memory units ${ }^{3,4}$, which has increased the strong demand for acceleration of AI computing by using special hardware ${ }^{5}$.

Among various AI accelerators, including present digital and analog AI accelerators built with silicon complementary metal-oxide semiconductor (CMOS) technologies ${ }^{6}$, a two-dimensional (2-D) array of memristors is one of the promising devices for area- and power-efficient edge-AI 
devices because of their resistive and nonvolatile properties and highly parallel analogue multiply-accumulate (MAC) fashion ${ }^{7}$. Memristors at a glance are similar to classical twoterminal resistors, but the resistance can be modified by applying voltage across the terminals, whereas it is retained when the magnitude of the voltage is sufficiently small ${ }^{8}$. These resistive and nonvolatile properties are quite suitable for implementing artificial synapses having resistive and long-term analog memory properties in ANNs, when the memristors are placed at the crossing points of two perpendicularly crossing nanowires ${ }^{9}$. This structure is referred to as a 2-D crossbar. Their early implementations were limited to small-scale crossbars, e.g., a 6×9-memristor crossbar for pattern classification ${ }^{10}, 32 \times 32$-memristor crossbar for sparse representation and pattern matching ${ }^{11}, 128 \times 64$-memristor crossbar for recurrent neural networks implementing long-short term memory (LSTM), and, recently, large-scale (2.5M) memristor crossbars for LSTM ${ }^{12}$ and a reconfigurable crossbar architecture of $4 \mathrm{M}$ memristors ${ }^{13}$ have been fabricated and demonstrated.

As 2-D memristor crossbars are targeted to accelerate AI computing, there exists a large structural difference between biological neural networks in the brain and the crossbars. Indeed, the brain has a three-dimensional (3-D) structure, i.e., neural tissues are structured in 3-D space. From the early stage of neuronal development, necessary neuronal wires, such as axons, dendrites, and synapses and their networks are grown as a result of natural unsupervised and supervised learning through their environment. On the other hand, 2-D memristor crossbars are optimized for fully connected (and hence, for unstructured) ANNs where all the possible synaptic connections between input and output neurons, i.e., memristors and wiring crossbars, are pre-placed upon device fabrication, which results in a significant increase in unused memristors as a result of learning, especially in structured $\mathrm{ANNs}^{14}$.

Here, we present a wetware prototype of a molecular synaptic medium where organic polymer wires are grown in monomer solution only if necessary, and demonstrate growth of synaptic networks on the medium. Recently, various organic synaptic devices have been reported in terms of different switching mechanisms and specialized benefits, e.g., low-power consumption $^{15}$ and 3-D building ability ${ }^{16,17}$. We employ a conducting polymer, poly(3,4ethylenedioxy-thiophene) doped with poly(styrene sulfonate) anions (PEDOT:PSS). It has attracted significant attention recently because of its wide variety of functionality, e.g., high conductivity $^{18}$, high thermoelectric conversion ${ }^{19,20}$, and high chemical sensitivity with bioadaptability $^{21,22}$, transparency, flexibility, and high environmental durability. Recently, their applications to elementary nonvolatile synapses and their 2-D array have also been 
demonstrated $^{15,23}$; however, as with 2-D memristor crossbars, they are not a space-free medium, as all the synapses between inputs and outputs are pre-placed. Although growth of wire-shaped conducting polymers has been reported, where their possible applications to information processing have naively been suggested ${ }^{24-27}$, their concrete application has not been shown yet. In this study, we show the concrete application of conductive polymer wires to an ANN where the growth process is directly linked to the learning process of artificial synapses. We first demonstrate an elementary ANN called a simple perceptron trained by linearly separable datasets (Boolean functions) implemented in our molecular-electronic hybrid ANN system consisting of the molecular synaptic media and their electronic controllers. We then present an ANN called an autoencoder performing unsupervised data-encoding (feature-extraction) tasks implemented in our system, and demonstrate that $3 \times 3$ ( 9 pixels) binary letters are successfully compressed into 3-bit code. No polymer wire exists before the learning, unlike 2-D memristor crossbar devices, but for now, necessary PEDOT:PSS wires are developed between electrodes (corresponding to artificial neurons) through the learning process. Consequently, a significantly small number of synapses (PEDOT:PSS wires) always developed, as compared to the number of contributable synapses in computer-simulated autoencoders having fully connected network structures. The complementary replacement of poorly grown polymers with better ones arose because of nonlinearity and variability upon updating the weights, which eventually resulted in a variabilitytolerant synaptic device based on the natural selection. As extending our 2-D synaptic media to 3-D is possible in principle, as shown in Supplementary Information Figure S6, the proposed methodology will provide possible solutions to the present problem called "Missing One Dimension" upon implementing 3-D neural networks on a 2-D chip ${ }^{28}$.

\section{Method}

Through electropolymerization, PEDOT wires are grown between electrodes immersed in EDOT [3,4-ethylenedioxythiophene] solution by applying bipolar alternate current (AC) voltage to the electrodes. It has been reported that a single and straight conducting polymer wire grows between electrodes with the sharp apex immersed in monomer solution when the gap distance is short and absolute bipolar square-wave AC voltage with a $50 \%$ duty cycle is applied ${ }^{25}$. We conducted an investigation in order to perform the wire growth of PEDOT, where PEDOT growth with aprotic electrolytes, PSS, $\mathrm{LiClO}_{4}$, BMIPF6, TBAPF6, and p-Ts were also attempted, and the PEDOT:PSS gave the best controllability and reproducibility with the thin shape of the wires. Precursor 
solution was prepared containing $0.135 \mathrm{M}$ EDOT and $0.02 \mathrm{M}$ PSS in acetonitrile and ultrapure water ratio of 1:1. Liquid EDOT and PSS solution were obtained from Sigma-Aldric. Electrodes reported in Fig. 1-3 were fabricated by the conventional photolithography technique. $\operatorname{Cr}(10 \mathrm{~nm})$ and $\mathrm{Au}(190 \mathrm{~nm})$ were deposited on a glass substrate by using EB1100 (Canon Anelva). The electrode array tip reported in Fig. 4 is composed of a sputtered $\operatorname{Cr}(50 \mathrm{~nm}) / \operatorname{Pd}(40 \mathrm{~nm}) / \mathrm{Au}(500$ $\mathrm{nm})$ layer and $\mathrm{Au}(500 \mathrm{~nm})$ plating film on a Pyrex glass substrate and a top coat polyimide film of 1- $\mu \mathrm{m}$ thickness. A solution trough made of polydimethylsiloxane was placed on the substrates with electrodes and filled with EDOT solution. The bipolar AC voltage with a square shape was generated using the arbitrary waveform generator WF1973 connected to a high-speed bipolar amplifier HSA4101 (NF Corporation). The AC voltage was applied to one side of the electrode, whereas the opposite electrode was connected to the grounded state (GND). Growing wire images were observed by the inverted optical microscope Olympus IX73 or metallurgical microscope KEYENCE VHX-500.

\section{Results and Discussion}

\section{Electropolymerization of PEDOT:PSS}

Figure 1a illustrates our setup where a bias electrode (left) is connected to an AC voltage source $\left(V_{\mathrm{s}}\right)$, and the other (right, ground electrode) is connected to the ground. This setup results in the directional polymerization of the monomer EDOT into PEDOT (see Method section for the experimental details). Here, we employ PSS, being a dopant of the formed PEDOT wires, as an electrolyte in monomer solution. Figure 1b shows a PEDOT:PSS wire bridging tips of bilateral triangular electrodes. No wire exists initially; however, applying $V_{\mathrm{s}}$ to the bias electrode initiates growth of the microwires from both tips of the electrodes toward the center, and eventually, the wires contact each other. The long-distance growth of PEDOT:PSS wires frequently induces dendritic branching of the wires, as shown in Fig. 1c. Among various waveforms of AC voltages, the bipolar alternative voltage with a square waveform $V_{\mathrm{s}} \equiv V_{\mathrm{a}} \cdot \operatorname{sgn}\left(\sin \left(2 \pi f_{\mathrm{s}} t\right)\right)+V_{\text {offset }}$, where $V_{\mathrm{a}}, \operatorname{sgn}(\cdot), f_{\mathrm{s}}, t$, and $V_{\text {offset }}$ represent the amplitude, sign function $(\in\{-1,0,1\})$, frequency, time, and direct current (DC) offset voltage, respectively, was employed for effective wire growth. In this study, $f_{\mathrm{s}}$ and $V_{\mathrm{a}}$ were set at $0.3-100 \mathrm{kHz}$ and $4-20 \mathrm{~V}$, respectively. Bipolarity of $V_{\mathrm{s}}$ is very important to the nature of wire growth because polymerization initiates from a dominant anodic electrode only. As shown in Fig. 1a, as the polarity of the electrodes (anode and cathode) is alternatively exchanged by $V_{\mathrm{s}}$, when $V_{\text {offset }}$ is set at the ground potential, symmetric wire growth initiated from both the electrodes will be observed. Negative and positive 
$V_{\text {offset }}$ will induce asymmetric wire growth initiated from the dominant anodic electrode only. When $V_{\text {offset }}$ is negative, the ground electrode becomes the dominant anodic, and wire growth is initiated from the ground electrode, whereas the bias electrode becomes the dominant anodic when $V_{\text {offset }}$ is positive, which results in wire growth from the bias electrode. Further, the nonzero $V_{\text {offset }}$ increases the number of branches upon wire growth. Increasing $\left|V_{\text {offset }}\right|$ eventually terminates the wire growth, which gives rise to polymerization at the entire surface of the anodic electrode.

We found a clear frequency dependence of growth rate and diameters of the PEDOT:PSS wires. Figure $1 \mathrm{~d}$ shows PEDOT:PSS wires grown by different $f_{\mathrm{s}}$, where low $f_{\mathrm{s}}$ induced thick and slow wire growth (Fig. 1d left), whereas high $f_{\mathrm{s}}$ resulted in thin and fast wire growth (Fig. 1d right). Figure 1e summarizes the dependence of the wire diameter on $f_{\mathrm{s}}$ ranging from $100 \mathrm{~Hz}$ to $2 \mathrm{MHz}$, where the wire diameter and $f_{\mathrm{s}}$ has a following relationship;

$$
\operatorname{diameter}(\mu m)=98 / \sqrt{f_{s}}
$$

This relationship indicates that the wire thickness, i.e., cross-section of wire, area of circle, is proportional to the wavelength, $1 / f_{s}$. Appling the square-wave AC voltage with a $50 \%$ duty cycle means that the anodic DC voltage is applied for half of the wavelength at the front edge of the growing wire. We consider that lengthwise polymerization arises at the moment of polarity $V_{\mathrm{s}}$ switch so that the growth speed depends on the frequency. Subsequent polymerization in lateral direction during static potential difference $V_{\mathrm{a}}$ is kept makes wire thicker.

We also found that i) $V_{\mathrm{a}}$ had an influence on the growth rate, i.e., a higher $f_{\mathrm{s}}$ required a larger $V_{\mathrm{a}}$ to maintain tolerable growth rates of wires in the experiments, and that ii) the number of grown wires was increased by increasing $V_{\mathrm{a}}$. At the beginning of the growth process, a single wire or a few wires were grown. When thick wires grown from both the electrodes were contacted (the joint conductance ranged from several tens of $\mu \mathrm{S}$ to several hundreds of $\mu \mathrm{S}$ in our experiments), further wire growth initiated from the electrodes was attenuated because spreading currents in the solution converged on the grown wire. On the other hand, when thin wires were contacted (the spreading current was not fully converged on the thin wires), the subsequent wires were successively grown between the electrodes. Figures $1 \mathrm{f}, 1 \mathrm{~g}$, and $1 \mathrm{~h}$ show images of grown wires taken after around 15 min of growth for each when $V_{\mathrm{a}}=7.5,10$, and $15 \mathrm{~V}$, respectively, with common $f_{\mathrm{s}}(20 \mathrm{kHz})$. The subsequent increase in $V_{\mathrm{a}}(>15 \mathrm{~V})$ gave rise to $\mathrm{H}_{2}$ gas bubbling at 
the dominant cathodic electrode because of a small nonzero $V_{\text {offset }}$, which resulted from electrolysis of the water solvent. The further increase in $V_{\mathrm{a}}$ caused bubbling at both the electrodes because the magnitudes of $V_{\text {offset }}$ became relatively small compared to the increased $V_{\mathrm{a}}$. Gas generation was abruptly observed when the number of wires was increased.

The conductance between electrodes increases with the wire growth. We can stop wire growth at any given time and the grown wire would keep its shape and conductance steadily; therefore, they can be used as resistive change memory. We measured the current flows through the wire by applying a small DC $(0.1 \mathrm{~V})$ voltage, avoiding polymerization. Oxidation potential of monomer EDOT is ca. $-0.8 \mathrm{~V}$. In our experiment, test voltage larger than $0.4 \mathrm{~V}$ made grown wires thicker after many number of measurement cycles. The electric circuit used for the measurement is explained in the Method section. Figure 1i shows the conductance increase between electrodes with the $250-\mu \mathrm{m}$ gap with a growth voltage of $10 \mathrm{kHz}$ and $40 \mathrm{~V}_{\mathrm{pp}}$ for $1 \mathrm{~s}$ in a cycle of growth. First, an abrupt increase in conductance occurs after 60 cycles because of the first wire's contact. The conductivity of PEDOT:PSS wire grown in this way was $0.01-1 \mathrm{~S} / \mathrm{cm}$ without wire diameter dependence. The first and second wire connection shows a stepwise increase, while the subsequent increase is continuous, because the multiple wires start multiple connections, attaching to each other. The conductance maximum is determined according to a saturation of the number of wires or occurrence of $\mathrm{H}_{2}$ gas bubbling that disrupts the wirings physically. The breakout condition of the $\mathrm{H}_{2}$ gas complexity depends on the combination of $\mathrm{V}_{\mathrm{pp}}$ and frequency of the applied AC voltage and shape of the electrodes.

The optimal condition, which favors the progress of the machine learning process, is continuous, and there is a linear conductance increase in a wider range. Figure $1 \mathrm{j}$ shows the wire growth between electrodes with a round shape apex of curvature radius of $50 \mu \mathrm{m}$, gap length of $50 \mu \mathrm{m}$, and $100-\mathrm{kHz}$ AC square voltage with $25 \mathrm{~V}_{\mathrm{pp}}$. The conductance increases continuously and attains a saturated conductance of $17 \mu \mathrm{S}$. The inserted image shows a large number of wires with high density, where it is difficult to see the wire because its diameter is about $0.2 \mu \mathrm{m}$. No wire grows between the wider electrode with the larger curvature radius of $100 \mu \mathrm{m}$ and gap length of $50 \mu \mathrm{m}$ until the $\mathrm{V}_{\mathrm{pp}}$ is increased over $40 \mathrm{~V}$, which causes $\mathrm{H}_{2}$ gas bubbling.

The wire grows directionally along the electrical potential gradient in solution. The primary wire generally grows close to the shortest path between the apex of electrodes. The subsequent wire growth frequently shows an elongation along the outer line of electrical force, as seen in Figs. If 
and 1i. They eventually move and fall into the center wire fascicle, as shown in Figs. $1 \mathrm{~g}$ and $1 \mathrm{~h}$. The drastic movement of the wire is considered due to the heat convection of solution raised from the electrical current flow. The high directivity of wire growth can be used to realize multiple wiring for plural electrodes. Figure $1 \mathrm{k}$ shows a wire sorting for three electrodes from one electrode controlling the respective conductance. The growth voltage applied to three plural electrodes was $20 \mathrm{kHz}$ and $25 \mathrm{~V}_{\mathrm{pp}}$ with a $0.1-\mathrm{V}$ offset, which advanced wire growth from the single counter electrode. A programmed Arduino controlled the mechanical relay of the plural electrode to attain the conductivity ratio, where the conductivity was set to 2,1 , and 5 for the top, middle, and bottom electrodes, respectively. After a wire connected to the middle electrodes, growth to the bottom and top electrodes conditioned and eventually attained an ordered ratio. The performance of multiple wiring is a peculiar competence of polymer wire growth. It is possible for the polymer wire to crosslink and grow between designated electrodes. Movies and images of the various polymer growths are shown in the Supplemental Information.

\section{Supervised learning - Simple perceptron for simple logic gate -}

An elemental function in learning is synapse plasticity, and the synapse component in a physical ANN structure is required to have a resistive change ability and memory function. Synapse weight in an ANN should both increase and decrease in accordance with the order from a learning program. The direction of the conductance change of PEDOT:PSS wire will only increase with the stiffening of the material. In a physical ANN configuration, synapse weight consists of two positive and negative elements, because the negative weight has the critical role of product-sum operation in an ANN. It is a general way to control the conductance of memristive devices in an only increasing manner through learning, and the decrease operation is used only when the saturated device must be reset. In our case, learning should complete before saturation of wire growth.

We preliminarily demonstrated simple logic AND and OR gates consisting of two input and one output neurons connected to two negative and positive synapses, as shown in Fig. 2a, trained by the simple perceptron algorithm. Four pairs of electrodes for polymer wire growth played the role of synapse. The four pairs of electrodes were connected to four electrical circuits, which were used to measure the conductance, as shown in Fig. 2b, where the circuit and relay are abbreviated comprehensibly. Test current flowing through the polymer wire was converted to voltage and read by the Arduino as synapse weights. Output results Y were calculated using Equation (1), 
mentioned in the Method section. The Arduino controlled the mechanical relay according to the installed simple perceptron program, shown in Fig. 2c.

As depicted in Fig. 3, the program starts and the TEST protocol switches all relay to test circuit. When the test circuit is connected, a DC test voltage of $1 \mathrm{~V}$ is applied. The Arduino reads serial output values at a time for all synapses, $1 \mathrm{p}, 1 \mathrm{n}, 2 \mathrm{p}$, and $2 \mathrm{n}$, and memorizes them as synapse weights of $\mathrm{w}_{1 \mathrm{p}}, \mathrm{w}_{1 \mathrm{n}}, \mathrm{w}_{2 \mathrm{p}}$, and $\mathrm{w}_{2 \mathrm{n}}$, respectively. Next is a random call of input $\mathrm{X}_{1}$ and $\mathrm{X}_{2}$ from four combinations of 1 and -1 , as shown in Fig. 3f. With the synapse weights and input, a perceptron's outputs $\mathrm{Y}$ are calculated as follows:

$$
\mathrm{Y}=\operatorname{sgn}\left\{\left(w_{1 \mathrm{p}}-w_{1 \mathrm{n}}\right) \mathrm{X}_{1}+\left(w_{2 \mathrm{p}}-w_{2 \mathrm{n}}\right) \mathrm{X}_{2}-\theta\right\}
$$

where $\operatorname{sgn}(x)$ is the sign function to split a product to 1 or -1 , i.e., if $x<0, \operatorname{sgn}(x)=-1$, and if $x \geqq 0, \operatorname{sgn}(x)=1 . \theta$ is the decision threshold, which has an effect on the learning progress, because the initial weight of physical synapses is not zero. A finite $\theta$ value was needed to prompt smooth progress of learning in an earlier stage. In the next step of the decision, if the outputs $Y$ are the same as the supervisee signal T, the YES flow goes back to new epoch. If $\mathrm{Y}$ is not $\mathrm{T}$, the NO flow goes to learning, where some synapses have to change their weight to become close to the correct answer. The training signal will be made by an error function as follows:

$$
\Delta \mathrm{W}_{i}=\varepsilon(\mathrm{T}-\mathrm{Y}) \mathrm{X}_{\mathrm{i}},
$$

where $\varepsilon$ is a positive constant. For example, if the inputs $X_{1}$ and $X_{2}$ are 1 and -1 for the AND gate, $\mathrm{T}$ is -1 and $\mathrm{Y}$ is 1 . When $\varepsilon=0.05$, the training functions, $\Delta \mathrm{W}_{1}$ and $\Delta \mathrm{W}_{2}$, are -0.1 and +0.1 , which indicates that synapse 1 should decrease by 0.1 and synapse 2 should increase by 0.1 of their weight, respectively. For the polymer case, an order of the synapse weight update is converted to an order to control mechanical relay. In this case, the Arduino program makes the order to make a connection to the AC growth power supply and GND for only the synapses of $1 \mathrm{n}$ and $2 \mathrm{p}$. The relays for the other half of the synapse without growth order and synapse with the right answer, $\Delta \mathrm{W}=0$, maintain the neutral position. After the learning is completed, the decision of $\mathrm{Y}=\mathrm{T}$ always becomes YES. Therefore, the test is repeated but no further learning order is commanded. We can stop the program running sooner or later.

Figure $2 d$ shows typical conductance changes through learning of the AND gate. During the learning phase, an $\mathrm{AC}$ voltage of $25 \mathrm{kHz}$ and $30 \mathrm{~V}_{\mathrm{pp}}$ for $1 \mathrm{~s}$ was applied. During the test phase, a $\mathrm{DC}$ voltage of $0.5 \mathrm{~V}$ for $0.5 \mathrm{~s}$ was applied and the current was measured. The conductance of $\mathrm{w}_{1 \mathrm{p}}$ abruptly increases to $0.3 \mu \mathrm{S}$ at the $130^{\text {th }}$ epoch, but $2 \mathrm{p}$ exhibits no wire bridging for a while. The 
$\mathrm{w}_{2 \mathrm{p}}$ abruptly increases to $0.6 \mu \mathrm{S}$ and $\mathrm{w}_{1 \mathrm{p}}$ subsequently increases to $0.7 \mu \mathrm{S}$ at around the $290^{\text {th }}$ epoch. After the polymer bridging in both $1 \mathrm{p}$ and $2 \mathrm{p}$ pairs of electrodes was attained, output results $\mathrm{Y}$ for every input combination yielded the correct answer against the supervised signal $\mathrm{T}$ of the AND gate. A pair of single wires or a few polymer wiring attained their logic functions by maintaining a balance of synapse weight. We performed the learning for the OR, NAND, and NOR as well, which are shown in Supplemental Information.

\section{Unsupervised learning - Autoencoder for feature extraction -}

An autoencoder is an unsupervised learning algorithm of an ANN, which learns a representation for a set of data, typically reducing the data dimensionality. The encoded data has its abstract feature extracted from the original high-dimensional data. Recently appeared powerful AI have involved autoencoders inside of their deep neural networks, so the autoencoder concept has become more widely used for learning. The autoencoder generally comprises a hidden layer between the encoder and decoder, as shown in Fig. 3a. The encoder compresses inputted data $V_{i}$ through a synapse network of $\mathrm{w}_{\mathrm{ij}}$ to a low-dimensional dataset of $\mathrm{h}_{\mathrm{j}}$. The decoder decompresses the data set of $h_{j}$ through the same synapse network of $w_{i j}$ to the new dataset of $V_{\text {di }}$. Mandatory of the autoencoder algorithm is the self-replication of $V_{d i}$ from $V_{i}$ by changing the synapse weight $\mathrm{w}_{\mathrm{ij}}$. If the inputted $\mathrm{V}_{\mathrm{i}}$ and outputted $\mathrm{V}_{\mathrm{di}}$ are not same, the only erroneous $\mathrm{w}_{\mathrm{i}} \mathrm{S}$ are updated, so no supervised data is needed, as shown in Fig. $3 \mathrm{~b}$. The learning is complete when all $\mathrm{V}_{\mathrm{di}}$ becomes identical to $\mathrm{V}_{\mathrm{i}}$ for all input databases.

We tried to make a polymer ANN that realizes feature extraction of three $3 \times 3$ binary letters into 3-pixel codes, learned in an autoencoder manner. The ANN consists of 9 input neurons and 3 hidden neurons, where a hidden neuron should comprise positive and negative synapses; therefore, the ANN require 54 synapses. The autoencoder built of an $9 \times 6$-polymer ANN used 9 substrates with 6 pairs of electrodes, which correlate with a single input neuron $V_{i}$ and 6 hidden neurons $h_{j \pm}$, as shown in Fig. 3c. The 9 divided terminals of the respective hidden neurons $\mathrm{h}_{\mathrm{j} \pm}$ are shortcircuited outside. Each of the $9 V_{i}$ neurons and 6 hidden $h_{i \pm}$ neurons are connected to respective electrical circuit boards, consists of four relays and Op amp based I/V convertor and only one terminal to electrode for polymer as shown in Fig. 3d. Total 15 boards are connected to each neuron of $\mathrm{V}_{\mathrm{i}}$ and $\mathrm{h}_{\mathrm{i}}$. The autoencoder algorithm has two test phases. One is encoding to work-out the hidden neuron $h_{i}$ with $V_{i}$ input; the other is the decoding process to work-out the output neuron $V_{\text {di }}$ with $h_{i}$ input, as shown in Fig. $3 b$. 
At the first test phase, positive and negative test DC voltage, $+0.1 \mathrm{~V}$ or $-0.1 \mathrm{~V}$ correlating with binary data of a letter, $\mathrm{X}, \mathrm{H}$ or $\mathrm{T}$, were applied to $\mathrm{V}_{\mathrm{i}}$ for $0.1 \mathrm{~s}$, where, the binary signal 1 or -1 correlates with the black and white colour, positive and negative test voltage, respectively. Here, the system measure the current flows into $h_{j^{+}}$and $h_{j-}$ at a time, which correlate with a result of sum of products $\sum_{i}^{9}\left(w_{i j+} V_{i}\right)$ and $\sum_{i}^{9}\left(w_{i j-} V_{i}\right)$, respectively. Encoded signal from nine-bit to three-bit at hidden neuron $h_{i}$ are calculated from the current values as follows.

$$
h_{j}=\operatorname{sgn}\left\{\sum_{i}^{9}\left(w_{i j+} V_{i}\right)-\sum_{i}^{9}\left(w_{i j+} V_{i}\right)-\theta\right\}
$$

Now hidden neuron $h_{j}$ should have any one data set of 3 pixel from 8 kinds shown in Fig. $3 a$ bottom. In the next test phase, test DC voltage $+0.1 \mathrm{~V}$ or $-0.1 \mathrm{~V}$ correlating with $\mathrm{h}_{\mathrm{j}}$ were applied to $h_{j \pm}$ and a current flow into neuron $V_{d i}$ were measured, where the neurons $V_{i}$ and $V_{d i}$ means single physical terminal $\mathrm{V}_{\mathrm{i}}$. Here, when $\mathrm{h}_{\mathrm{i}}$ is $+1,+0.1 \mathrm{~V}$ and $-0.1 \mathrm{~V}$ were applied for $\mathrm{h}_{\mathrm{i}^{+}}$and $\mathrm{h}_{\mathrm{i}}$, simultaneously, when $h_{i}$ is -1 , voltages of opposite polarity were applied. Measured current at a board connected terminal of neuron $\mathrm{V}_{\mathrm{i}}$ correlate with a result of sum of products $\sum_{j}^{3}\left(w_{i j} h_{j}\right)$. Decoded $\mathrm{V}_{\mathrm{di}}$ were calculated as follows.

$$
V_{d i}=\operatorname{sgn}\left\{\sum_{j}^{3}\left(w_{i j}\right)-\theta\right\}
$$

In the learning phase, when the $\mathrm{V}_{\mathrm{i}}$ and $\mathrm{V}_{\mathrm{di}}$ are not same, which synapse weight should be increased or decreased is decided by following error function;

$$
\Delta \mathrm{W}_{i j}=\epsilon\left(V_{i}-V_{d i}\right) h_{j}
$$

When the $\Delta \mathrm{W}_{i j}$ is not zero, terminals of the selected neuron $\mathrm{i}$ and $\mathrm{j}$ are connected to the $\mathrm{AC}$ growth voltage of $20 \mathrm{kHz}$ and $40 \mathrm{~V}_{\mathrm{pp}}$ and the GND for 1s, thus the polymer wire in $\mathrm{w}_{\mathrm{ij}}$ grows. The polarity of $\Delta \mathrm{W}_{i j}$ selects the learning terminal $\mathrm{h}_{\mathrm{i}+}$ or $\mathrm{h}_{\mathrm{i} . .}$. The rest terminals are floated. After the learning phase, two test phases start again. When the all $V_{i}-V_{d i}$ becomes zero in each epoch many times repeatedly, no weight update take place, so that means the learning complete.

A typical result of the autoencoder machine learning by using polymer wire growth for three 9pixel characters $\mathrm{X}, \mathrm{H}, \mathrm{T}$ is shown in Figure 3e. The upper panel shows the error rate, which is the ratio of the number of $\mathrm{V}_{\mathrm{i}} \neq \mathrm{V}_{\mathrm{di}}$ in 9 , and the lower panel shows the conductance change for all 54 synapses during learning. One learning epoch is one cycle for one data set. The three characters were input in series every three epochs. It is clear that the error rate decreases gradually as the learning proceeds, and they eventually converge to zero. The conductance of the polymer increases in a respective manner, showing small fluctuations. The start of the conductance 
increase is after 100 epochs because of the gap length between electrodes of $400 \mu \mathrm{m}$, as shown in Fig. 3c. The achieved network results encoded from $X, H$, and $T$ letters to $(1,-1,-1),(-1,-1,-$ $1)$, and $(1,1,1)$, respectively, where these combinations were incidentally determined through the learning.

Figure $3 \mathrm{f}$ shows the update order for wire growth indicated by a red line, and the resulting conductance change is indicated by the green color depth for all 54 synapses, where the learning epoch flows from top to bottom. The lower block in Fig. 3f shows the same update order and weight change of a synapse simulated in software for comparison. Whereas the polymer autoencoder has been converged after 500 learning epochs, the software converges the same autoencoder within about 30-60 learning epochs. This is because the single growth order for the polymer does not make the conductance increase, because of the noticeable nonlinearity of conductance increase against growth order, as shown in Fig. 1i. It was interesting that the polymer ANN consists of a small number of synapses, which has a significant weight of conductance. As shown in Figs. 3g and 3h, whereas the polymer ANN consists of almost 30 synapses with zero weight, the software simulated ANN always has less than 10 zero-weight synapses. It is considered that the complementary replacement of the poorly grown polymer with a better polymer arose because of the individual specificity of polymers resulting in a natural selective network consisting of a minimum requirement of wiring.

We made improvements to the machine learning system being poised to respond to increases in node number. Figure 4a shows an array of electrodes, which has a round shape with a gap length of $50 \mu \mathrm{m}$. The electrode array tip consists of 90 pairs of electrodes, of which the terminals are wire-bonded to hard wirings of the outside. The electrodes are covered by polyimide film, leaving the facing area bare, as shown in Fig. 4a; therefore, polymer growth for every pair of electrodes can be operated in a single solution trough at a time. The control electric board mounts a shift resistor to control 8 mechanical relays to the terminals. Only four signal lines from the Arduino can control all $8 \times N$ terminal relays on $N$ boards simultaneously, because the shift resistors are connected in series.

We performed an autoencoder machine learning of three $3 \times 3$ binary letters again by using this system. Seven boards for upstream and seven boards for downstream were used. All boards shared three inner circuit switching signals and used the same four signals to the series of shift resistors, 
and seven test-out terminals in the lower stream were connected to the read terminal in the Arduino. Eventually, the number of occupied terminals in the Arduino was only 17 even if the number of network synapses increased more, which is considerably decreased from the previous system occupying 60 terminals. In this case, all synapse weights and polymer resistances were measured to switch 8 times from terminal 1 to 8 simultaneously at every board with the application of a test DC voltage of $0.1 \mathrm{~V}$ to opposite electrodes. The measured conductance was assigned to all synapse weights $\omega_{i j}$, respectively. The $\mathrm{h}_{\mathrm{i}}$ and $\mathrm{V}_{\mathrm{di}}$ were derived by using the $w_{i j}$, immediately, in a manner of Equations (3) and (4) in the Method section. The update order for synapse weight determined by Equation (5) was randomly selected depending of the error rate. The update order was realized to connect the facing mechanical relay of designated pairs of electrodes to grow the polymer, where the upper stream connects to the growth $\mathrm{AC}$ voltage of $100 \mathrm{kHz}$ and $12 \mathrm{~V}_{\mathrm{p}-\mathrm{p}}$ and the lower stream connects to the GND.

Figures $4 \mathrm{c}$ and $4 \mathrm{~d}$ show optical microscope images of a typical polymer wiring after learning. They have respective conductance related to the number of polymers bridging between the electrodes. Figures $4 \mathrm{e}-\mathrm{f}$ show the error rate and conductance change during the learnings. The conductance increase starts within a few tens of learning epochs, and the learning completes within 100 epoch or less. Figure $4 \mathrm{f}$ shows the results of over-writings of $\mathrm{X}, \mathrm{H}$, and $\mathrm{T}$ after the learning of I, L, and P. The improved condition of the hard-wiring and improved electrode array and solution trough made the machine learning process for the polymer wire easy and reliable. The success probability of the autoencoder learning became nearly tenfold that of previous devices.

In comparison with other material-based techniques, the single learning epoch and entire term for learning completion in this technique are extremely long, i.e., the one epoch is almost 1.5 second and the number of epoch for learning were from one to few hundreds. The memristor device consisting of metal oxide layers ${ }^{10,11,29,30}$ can be operated by short epoch, e.g., one epoch is generally less than sub-milli-second and the number of learning epoch to complete similar task are in few tens. Silver atomic switch device ${ }^{31}$ seems to require relatively longer time period, e.g., order of second was needed to make memristive conductance change, though the switching can be operated in nano-second order. Organic memristive device ${ }^{15-17}$ require relatively longer time period than the order of milli-seconds to realize effective conductance change. Slower weight 
update ability in our polymer grows system is trade off relationship with the benefit of the spacefree growth ability, of which synaptic medium grows only when necessary.

It should be noted that the PEDOT:PSS wires bridging between electrodes can be dried and hold the resistance permanently in the ambient condition, though they will never be updated. Figures $4 \mathrm{c}$ and $4 \mathrm{~d}$ show polymer wires after drying, maintaining the bridging. The conductance of the dried wire slightly decreases with the weight difference of less than $5 \%$ than that in solution. We confirmed that a dried wire maintained the same conductance in ambient conditions for 6 months. We still have the problem of wire breaking due to capillary phenomena in the drying process arising in $10 \%$ of pairs of electrodes. It is expected to be solved by improving the drying process or resolving the use in a solution cell. Machine learning using polymer wire growth does not require expensive equipment and extreme conditions. A resistance array of polymer wires is a preferable candidate of a learnable tip with arranged multi resistances to adopt tasks in future neuromorphic hardware.

The wire directionally grows along the electrical potential gradient, so it can connect between designated terminals in 3-D free space in principle, though the polymer wire shown in this study basically grew on a 2-D bottom plane of solution. We tried polymer wiring in 3-D space using sharpened metal tips and buried metal terminals as well. Wiring was easily attained; however, the conductance control of the 3-D multi-branching was very difficult to perform (see Supplemental Information). It is considered that the multi-terminals we made were too large. Based on the condition we investigated, a challenge to overcome was fabricating multi-electrodes with a small area contacting solution, e.g., $10^{2}-100^{2} \mu \mathrm{m}^{2}$, with a fine pitch of 50-400 $\mu \mathrm{m}$, where they faced each other at a distance of 50-400 $\mu \mathrm{m}$. A small metal-exposing area is effective in making a sharp pathway of electrical potential gradient. The right ratio of pitch and gap length is needed to branch the wires connecting a number of vicinal counter electrodes. Finely fabricated multiterminals should make the 3-D polymer wiring possible.

\section{Conclusions}

We have investigated PEDOT:PSS wire growth between electrodes in monomer solution and attained good conditions to control its resistance change that can be used as resistive change memory. A machine learning system specialized for polymer wire growth was developed and performed learnings to form an ANN synaptic weight arrangement consisting of polymer wires. 
The resistance array of polymer wires showed a high capability for synapse weight update by increasing their number of wires bridging between electrodes. Autoencoder learning that enables feature extraction of three 9-pixel binary data into 3-pixel data was successfully demonstrated by using the 54-polymer synapse on an electrode array tip. Because of the high durability of the material, the learned resistance array of the PEDOT:PSS polymer wires could be used as a learnable tip to adopt a task in future neuromorphic hardware. The developed technique has machine learning versatility for various materials that can change its resistivity by introducing external voltage or current. In the near future, an on-demand and easy-to-use approach for the learning of neuromorphic hardware for the end user will be proposed. This work broadens the variety of candidate materials and frameworks to create physical neuromorphic hardware. 


\section{Figure caption}

Fig. 1 PEDOT:PSS polymer wire growth in EDOT monomer solution. a Simple schematic of experimental setup and molecular structural formula. $\mathbf{b}-\mathbf{d}$ and $\mathbf{f}-\mathbf{h}$ Optical microscope images of grown PEDOT:PSS wire between electrodes in EDOT solution by applying bipolar AC voltage with square shape. b Bipolar AC voltage of $300 \mathrm{~Hz}$ and $8 \mathrm{~V}_{\mathrm{pp}}$ for electrodes with gap of $150 \mu \mathrm{m}$. c Bipolar AC voltage of $300 \mathrm{~Hz}$ and $10 \mathrm{~V}_{\mathrm{pp}}$ for electrodes with gap of $600 \mu \mathrm{m}$. d Bipolar AC voltage with voltage offset of $0.1 \mathrm{~V}$, of which the frequency increased from 300 to $50 \mathrm{kHz}$ and $\mathrm{V}_{\mathrm{pp}}$ also increased from 8 to $10 \mathrm{~V}$, was applied for electrodes with a gap of $150 \mu \mathrm{m}$. e Frequency dependence on diameter of PEDOT:PSS wire. $\mathbf{f}-\mathbf{h}$ Time series of wire growth by applying AC voltage of $20 \mathrm{kHz}$ at 15,20 , and $30 \mathrm{~V}_{\mathrm{pp}}$, respectively. Images were taken after 5 minutes from the voltage application or increase. $\mathbf{i}$ and $\mathbf{j}$ Conductance change between electrodes with polymer growth by applied AC voltage of $\mathbf{i} 10 \mathrm{kHz}$ with $40 \mathrm{~V}_{\mathrm{pp}}$ for electrodes with $250-\mu \mathrm{m}$ gap and $\mathbf{j} 100$ $\mathrm{kHz}$ with $25 \mathrm{~V}_{\mathrm{pp}}$ for electrodes with 50- $\mu \mathrm{m}$ gap with round shape, respectively. $\mathbf{k}$ Plural polymer wiring control branching to three different electrodes from single one by applied AC voltage of $20 \mathrm{kHz}$ and $25 \mathrm{~V}_{\mathrm{pp}}$ with $0.1-\mathrm{V}$ offset. The conductance ratio was set as 2:1:5 for top:middle:bottom electrodes.

Fig. 2 Machine learning of simple logic gate comprising wiring polymer. a Two input and one output single ANN layer, where a synapse consists of two positive and negative divided synapses. b Scheme of machine learning system for polymer wire growth, which consists of a substrate with four pairs of electrodes, four electrical circuits that can switch between growth or test mode, an AC power supply, DC power supply, and Arduino. c Flowchart of simple perceptron algorithm. A step of decision "Y equal to T or not" proceeds a learning epoch. d Optical microscope image of after AND gate learning for four pairs of electrodes and polymer wired between $1 p$ and $2 p$ pairs of electrodes. e Conductance change during AND gate learning. $\mathbf{f}$ Reference chart of input $\mathrm{X}_{\mathrm{i}}$ and supervisor $\mathrm{T}$ for AND, OR, NAND, and NOR gate.

\section{Fig. 3 Unsupervised autoencoder learning by 54 synapses of polymer wiring. a} Representation of an autoencoder consisting of input neuron $V_{i}$, hidden neuron $h_{j}$, and output neuron $\mathrm{V}_{\mathrm{di}}$ forming two encode and decode $\mathrm{ANN}$ layers. Hidden neuron $\mathrm{h}_{\mathrm{j}}$ consists of positive and negative divided neurons. b Flowchart of autoencoder algorithm. $\mathbf{c}$ Grass substrates with 6 pairs of Au electrodes, which correlate to an input neuron $V_{i}$ and 6 hidden neurons $h_{j}$. The pairs 
of electrodes have a sharp apex with a gap of $250 \mu \mathrm{m}$. d Electric circuit board for autoencoder learning. e Error rate and conductance change of a successful autoencoder learning by polymer wire synapses, which can encode three 9-pixel characters (X, H, T) into respective 3-pixel data. $\mathbf{f}$ Upper block: update order for wire growth (red line) and conductance change for all 54 synapses (green color depth) shown with learning flow from top to bottom. Lower block: an example of same update order and weight change resulting in software autoencoder calculation. $\mathbf{g}$ and $\mathbf{h}$ Histogram of synapse weight that constitute accomplished autoencoder for $\mathbf{g}$ conductance of polymer wire and $\mathbf{h}$ numerical value in software.

Fig. 4 Machine learning system being poised to respond to increasing node number. a Electrode array tip for polymer wire growth consisting of 90 pairs of Au electrodes with $50-\mu \mathrm{m}$ gap with round shape. Wiring area is covered by polyimide film. b Schematic circuit diagram of an electric circuit board with simultaneous switching system to multi terminals. The photo shows the external appearance of the system consisting of 15 boards. $\mathbf{c}$ and $\mathbf{d}$ Typical optical microscope images of polymer wires bridging the electrodes, which were taken after drying. $\mathbf{e}$ and $\mathbf{f}$ Error rate and conductance change during autoencoder learnings for $\mathbf{e}(\mathrm{X}, \mathrm{Y}, \mathrm{Z})$ and $\mathbf{f}(\mathrm{I}, \mathrm{L}, \mathrm{P})$ and overwritten $(X, H, T)$.

\section{References}

1 Krizhevsky, A., Sutskever, I. \& Hinton, G. E. ImageNet classification with deep convolutional neural networks. Communications of the ACM 60, 84-90, doi:10.1145/3065386 (2017).

2 LeCun, Y., Bengio, Y. \& Hinton, G. Deep learning. Nature 521, 436, doi:10.1038/nature14539 (2015).

3 Laughlin, S. B., de Ruyter van Steveninck, R. R. \& Anderson, J. C. The metabolic cost of neural information. Nature Neuroscience 1, 36-41, doi:10.1038/236 (1998).

4 Horowitz, M. 1.1 Computing's energy problem (and what we can do about it). IEEE 10-14, doi:10.1109/ISSCC.2014.6757323 (2014).

5 Sze, V., Chen, Y.-H., Yang, T.-J. \& Emer, J. S. Efficient processing of deep neural networks: A tutorial and survey. Proceedings of the IEEE 105, 2295-2329, (2017).

6 Merolla, P. A. et al. A million spiking-neuron integrated circuit with a scalable communication network and interface. Science 345, 668-673, doi:10.1126/science.1254642 (2014). 

Materials 18, 309-323, doi:10.1038/s41563-019-0291-x (2019). Yang, J. J., Strukov, D. B. \& Stewart, D. R. Memristive devices for computing. Nature Nanotechnology 8, 13-24, doi:10.1038/nnano.2012.240 (2013).

$9 \mathrm{Li}$, C. et al. Long short-term memory networks in memristor crossbar arrays. Nature Machine Intelligence 1, 49-57, (2019).

10 Prezioso, M. et al. Training and operation of an integrated neuromorphic network based on metal-oxide memristors. Nature 521, 61-64, doi:10.1038/nature14441 (2015).

11 Sheridan, P. M. et al. Sparse coding with memristor networks. Nature Nanotechnology 12, 784-789, doi:10.1038/nnano.2017.83 (2017).

12 Tsai, H. et al. Inference of long-short term memory networks at software-equivalent accuracy using 2.5M analog phase change memory devices. Proceedings of the 2019 Symposium on VLSI Technology, (2019).

13 Mochida, R. et al. A 4M synapses integrated analog ReRAM based 66.5 TOPS/W neuralnetwork processor with cell current controlled writing and flexible network architecture. IEEE Symposium on VLSI Technology, (2018).

14 Mittal, S. A Survey of ReRAM-based architectures for processing-in-memory and neural networks. Machine Learning and Knowledge Extraction 1, 75-114, doi:10.3390/make1010005 (2019).

15 van de Burgt, Y. et al. A non-volatile organic electrochemical device as a low-voltage artificial synapse for neuromorphic computing. Nature Materials 16, 414-418, (2017).

16 Wu, C., Kim, T. W., Choi, H. Y., Strukov, D. B. \& Yang, J. J. Flexible three-dimensional artificial synapse networks with correlated learning and trainable memory capability. Nature Communications 8, 752, doi:10.1038/s41467-017-00803-1 (2017).

17 Lin, Y.-P. et al. Physical realization of a supervised learning system built with organic memristive synapses. Scientific Reports 6, 31932, doi:10.1038/srep31932 (2016).

18 Wei, Q., Mukaida, M., Naitoh, Y. \& Ishida, T. Morphological change and mobility enhancement in PEDOT:PSS by adding co-solvents. Advanced Materials 25, 2831-2836, doi:10.1002/adma.201205158 (2013).

19 Kim, G.-H., Shao, L., Zhang, K. \& Pipe, K. P. Engineered doping of organic semiconductors for enhanced thermoelectric efficiency. Nature Materials 12, 719-723, doi:10.1038/nmat3635 (2013). 

thermoelectric metrics in ultra-long electrodeposited PEDOT nanowires. Nano Letters 11, 125-131, doi:10.1021/nl103003d (2010).

21 Khodagholy, D. et al. In vivo recordings of brain activity using organic transistors. Nature Communications 4, 1573, doi:10.1038/ncomms2573 (2013).

22 Strakosas, X., Bongo, M. \& Owens, R. M. The organic electrochemical transistor for biological applications. Journal of Applied Polymer Science 132, doi:10.1002/app.41735 (2015).

23 Gkoupidenis, P., Koutsouras, D. A. \& Malliaras, G. G. Neuromorphic device architectures with global connectivity through electrolyte gating. Nature Communications 8, 15448, doi:10.1038/ncomms15448 (2017).

24 Das, A., Lei, C. H., Elliott, M., Macdonald, J. E. \& Turner, M. L. Non-lithographic fabrication of PEDOT nano-wires between fixed Au electrodes. Organic Electronics 7, 181-187, doi:10.1016/j.orgel.2005.12.006 (2006).

25 Thapa, P. S. et al. Directional growth of polypyrrole and polythiophene wires. Applied Physics Letters 94, 033104, doi:10.1063/1.3072611 (2009).

26 Kannan, B., Williams, D. E., Laslau, C. \& Travas-Sejdic, J. The electrochemical growth of highly conductive single PEDOT (conducting polymer):BMIPF6 (ionic liquid) nanowires. Journal of Materials Chemistry 22, 18132-18135, doi:10.1039/C2JM33107A (2012).

27 Koizumi, Y. et al. Electropolymerization on wireless electrodes towards conducting polymer microfibre networks. Nature Communications 7, 10404, doi:10.1038/ncomms10404 (2016).

28 Shibata T. A brain-mimicking VLSI system for human-like perception. Informatics 6 , in press.

29 Alibart F. et al. Pattern classification by memristive crossbar circuits using ex sith and in situ training. Nature Communications 4, 12072, doi:10.1038/ncomms3072 (2013).

30 Park S. et al. Electronic system with memristive synapses for pattern recognition. Scientific Reports 5, 10123, doi:10.1038/srep10123 (2015).

31 Ohno T. et al. Short-term plasticity and long-term potentiation mimicked in single inorganic synapses. Nature Materials 10, 591, doi:10.1038/NMAT3054 (2011). 
Figure 1
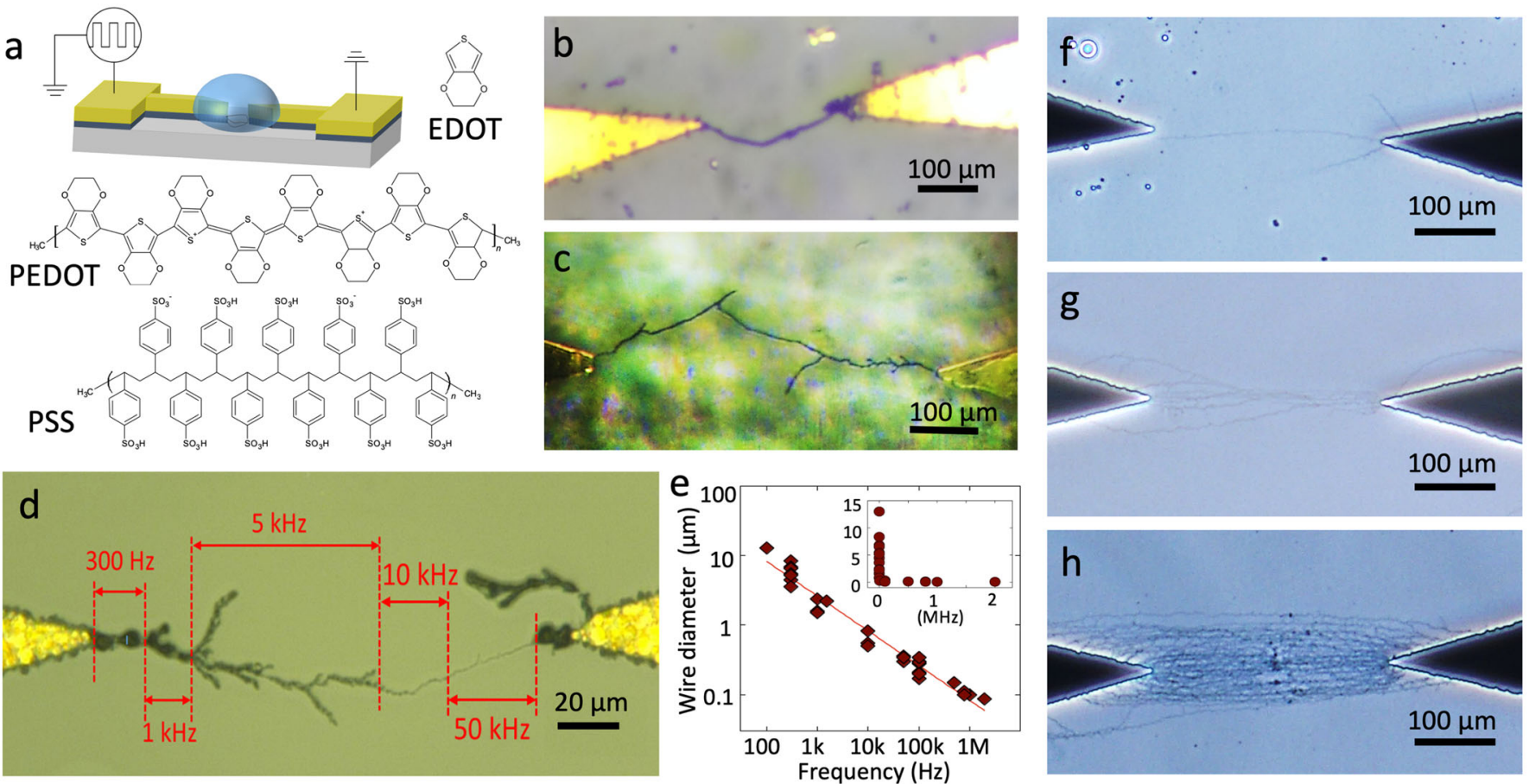

$100 \mu \mathrm{m}$
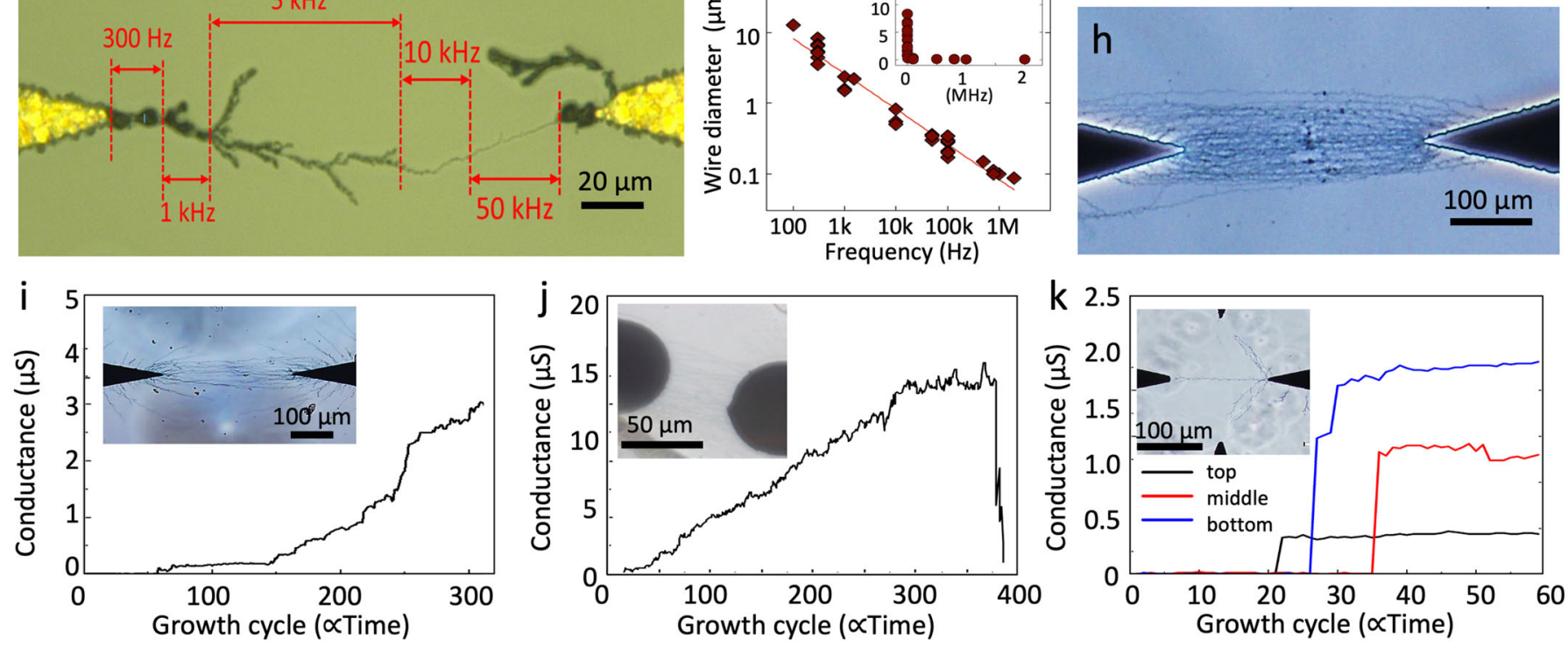
Figure 2
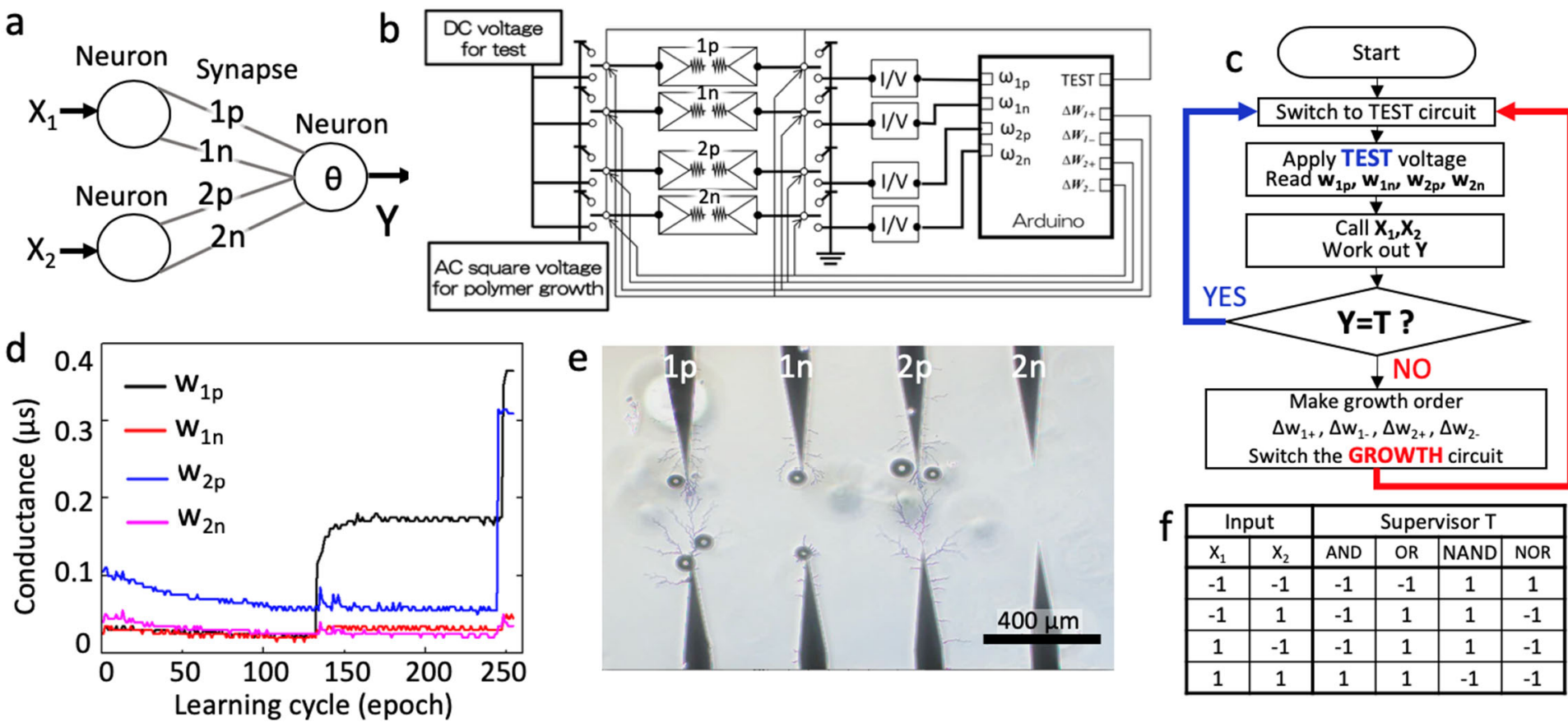
Figure 3

a

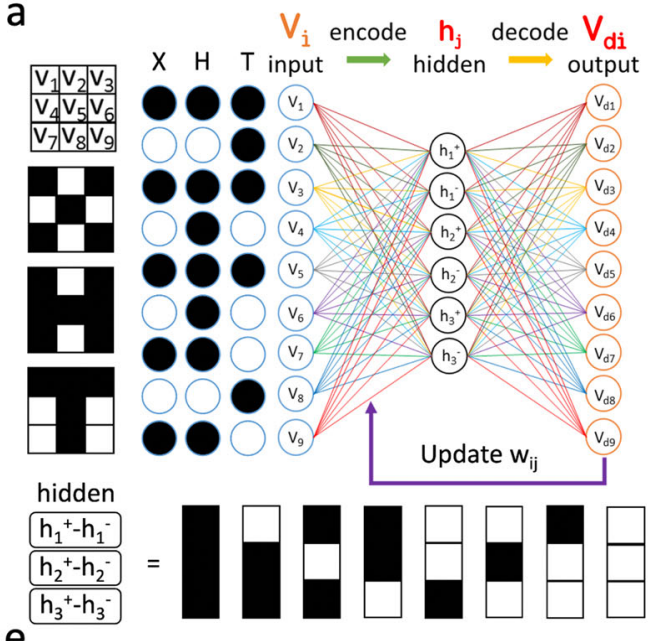$$
\text { e }
$$

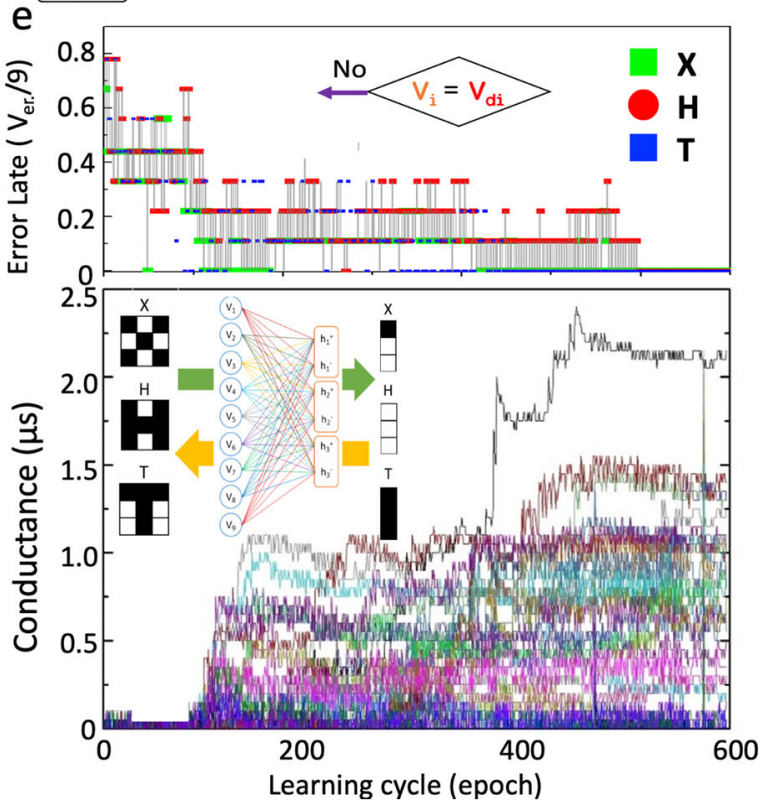

b

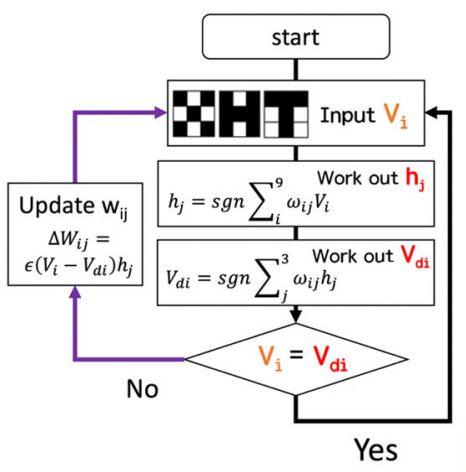

$f$

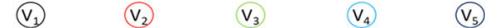

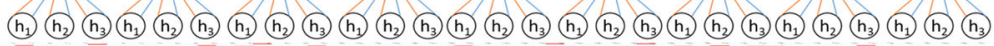
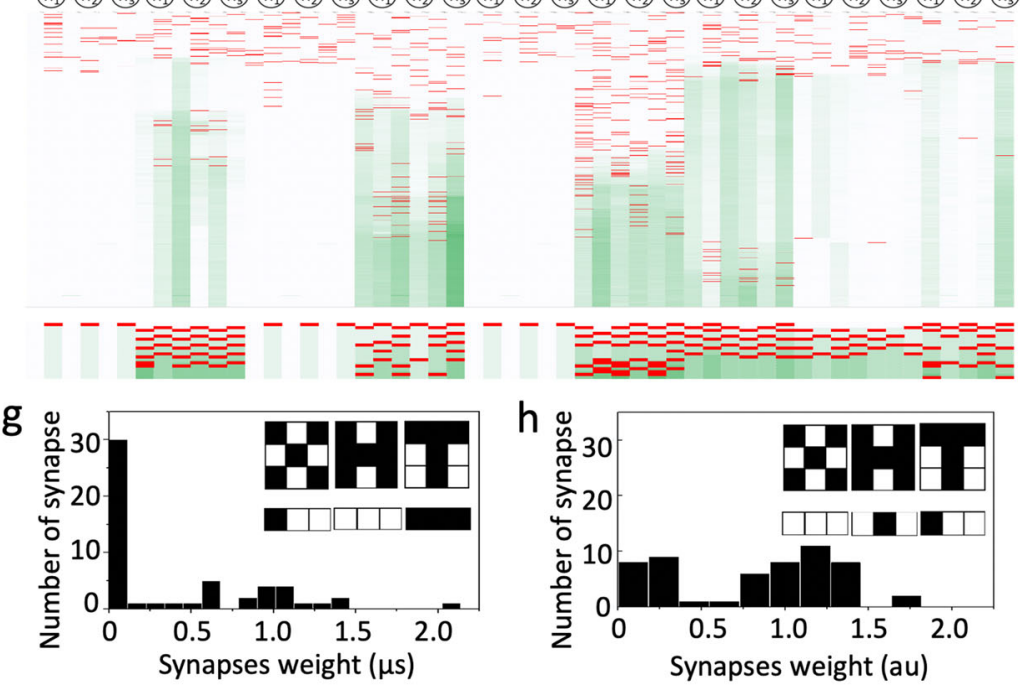
Figure 4

a
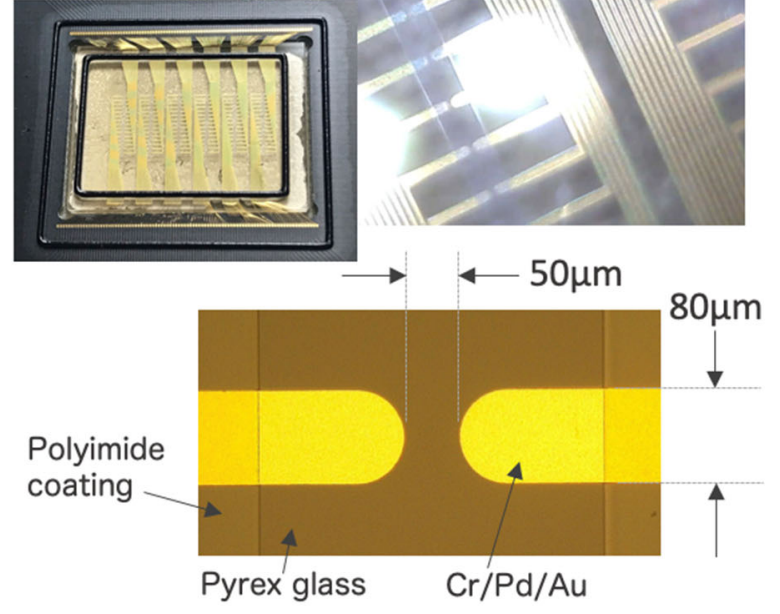

e

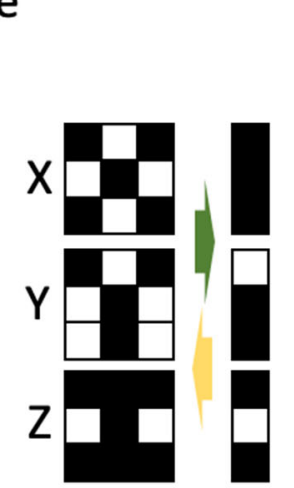

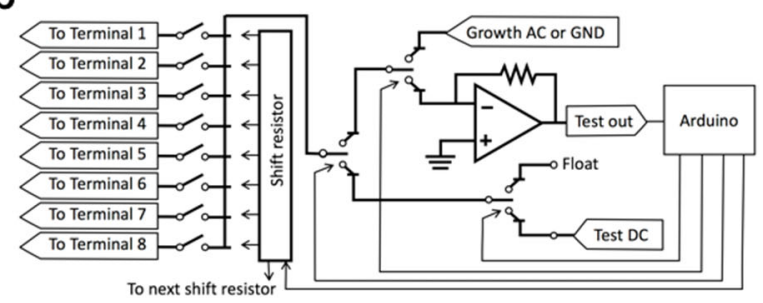
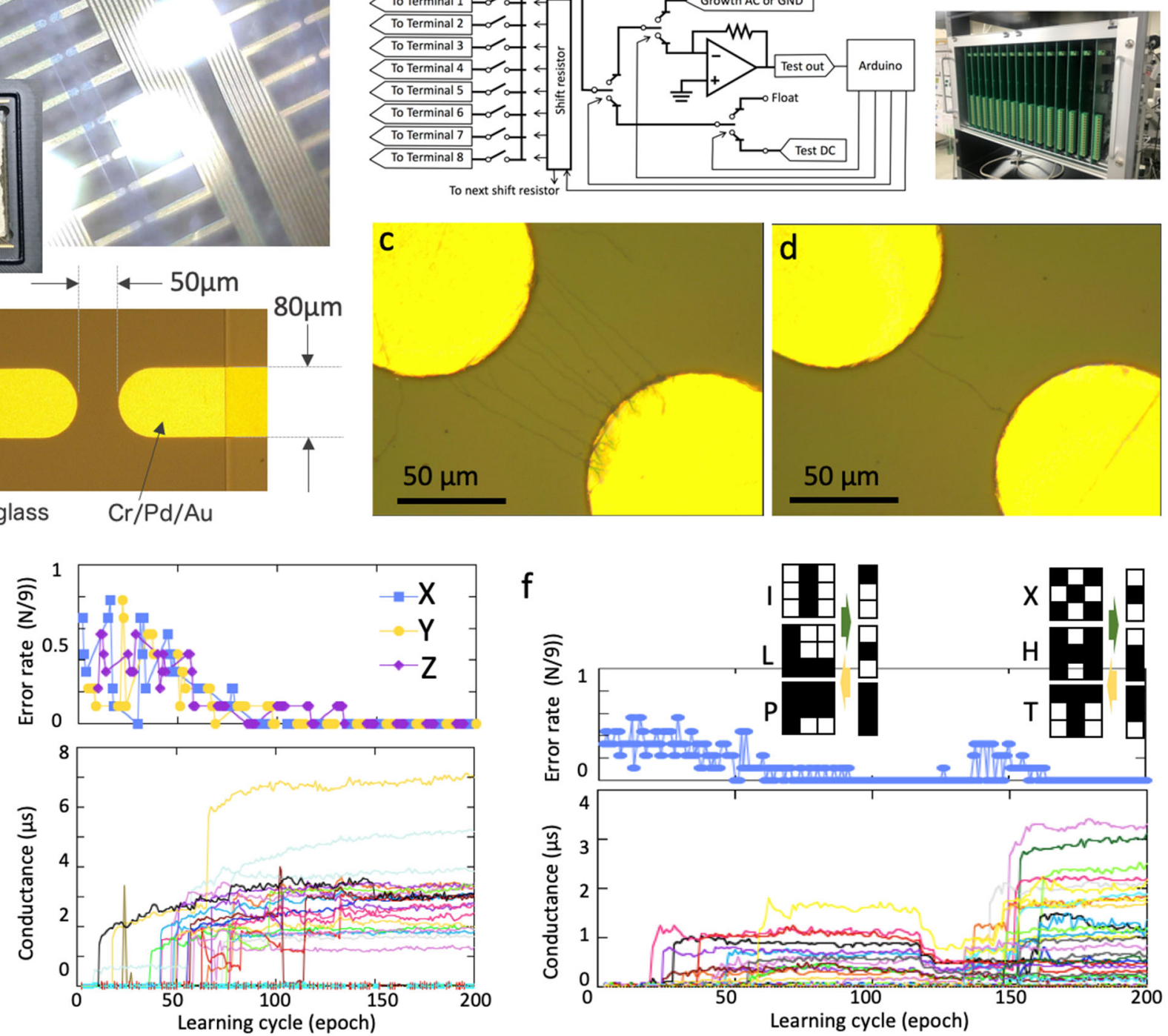